\title{
The use of the Developmental Coordination Disorder Questionnaire in Australian children
}

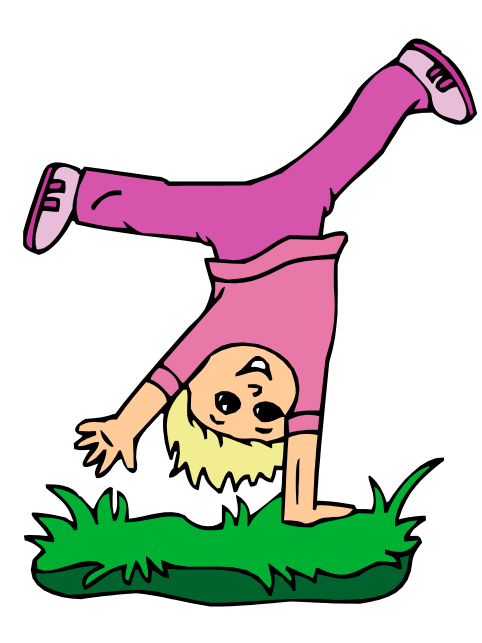

\section{Introduction}

The Developmental Coordination Disorder Questionnaire (DCDQ) is a parentrated questionnaire used for identifying children with Developmental Coordination Disorder (DCD) (Wilson, Kaplan, Crawford, Campbell, \& Dewey, 2000). This test was developed in Canada using clinically referred children between 8 and 14 years old. Green et al. (2005) and Schoemaker et al. (2003 cited in Green et al., 2005) have reported a low overall agreement between the DCDQ and other motor measures in identifying motor difficulties in European children. However, there has not been any reported validity study on the DCDQ using Australian children. Thus, the current study examined the accuracy of the DCDQ in identifying DCD in Australian children. The McCarron Assessment of Neuromuscular Development (MAND; McCarron, 1982) was used as a criterion measure to determine the presence or absence of DCD.

Aims of the study are to assess:

1. The concurrent validity of the DCDQ using the MAND

2. The discrimination and predictive accuracy of the DCDQ

\section{Method}

\section{Participants}

A total of 129 children were recruited from the primary schools within the Perth metropolitan area. There were 91 males and 38 females with age ranging from 9.62 years to 12.75 years $(\underline{M}=11.15, \underline{S D}=0.81)$. All participants were of normal to corrected vision, normal hearing, no neurological disorder, no current injury or permanent disability to their upper limbs. Among them, 32 children were previously diagnosed with a single or comorbid childhood disorders of ADHD, Learning disorder or motor problems.

\section{Measures}

- Developmental Coordination Disorder Questionnaire (DCDQ; Wilson et al. 1998). The DCDQ total score is made up of four factors scores, namely control during movement, fine motor/handwriting, gross motor/planning and general coordination. The DCDQ score of 0- 48 indicates DCD, 49- 57 indicates suspected DCD and 58- 85 indicates no DCD. The present study combined children with DCD and suspected DCD as a single DCD group.

- McCarron Assessment of Neuromuscular Development (MAND; McCarron, 1982). Children who have an NDI score of $\leq 85$ (below $15^{\text {th }}$ percentile) on the MAND were identified as having DCD and those $>85$ were considered as no DCD. The NDI range of 71 to 85 indicates a mild disability; 55- 70 indicates a moderate disability; and those below 55 as severe disability.

Procedure

The DCDQ was sent to the parents upon written consent and the completed questionnaire was returned to the researcher via mail. The MAND was administered to the child either in his/her school or at Curtin University.

\section{Results}

\section{Relationship between tests}

A Spearman rank order correlation analysis revealed a statistically significant but low association between DCDQ and MAND, rho $(127)=.37, p=.01$, sharing only $14 \%$ of the variance, suggesting that each test ranks the children's performance differently.

\section{Case agreement}

The degree of agreement between tests was low (Kappa $=0.284)$ with a proportion of agreement of 0.55 for the DCD cases (see Table 1 ).

\begin{tabular}{|c|c|c|}
\hline & DCD & $\begin{array}{l}\text { IDI) } \\
\text { non-DCD }\end{array}$ \\
\hline \multicolumn{2}{|l|}{ DCDQ } & $\begin{array}{l}22 \\
\text { (b) }\end{array}$ \\
\hline Non-DCD & $\begin{array}{l}20 \\
\text { (c) }\end{array}$ & $\begin{array}{l}63 \\
\text { (d) }\end{array}$ \\
\hline Total & 44 & 85 \\
\hline
\end{tabular}

Discrimination accuracy

The discrimination accuracy of the DCDQ was inferred from its sensitivity, specificity, and the positive and negative predictive values (see Table 2) using the formula recommended by Portney and Watkins (2000).

Table 2. Discrimination accuracy of the DCDQ with the MAND as criterion measure

\begin{tabular}{ll}
\hline Discrimination accuracy & DCDQ \\
\hline Sensitivity & $55 \%$ \\
Specificity & $74 \%$ \\
Positive predictive value & $52 \%$ \\
Negative predictive value & $76 \%$ \\
\hline
\end{tabular}

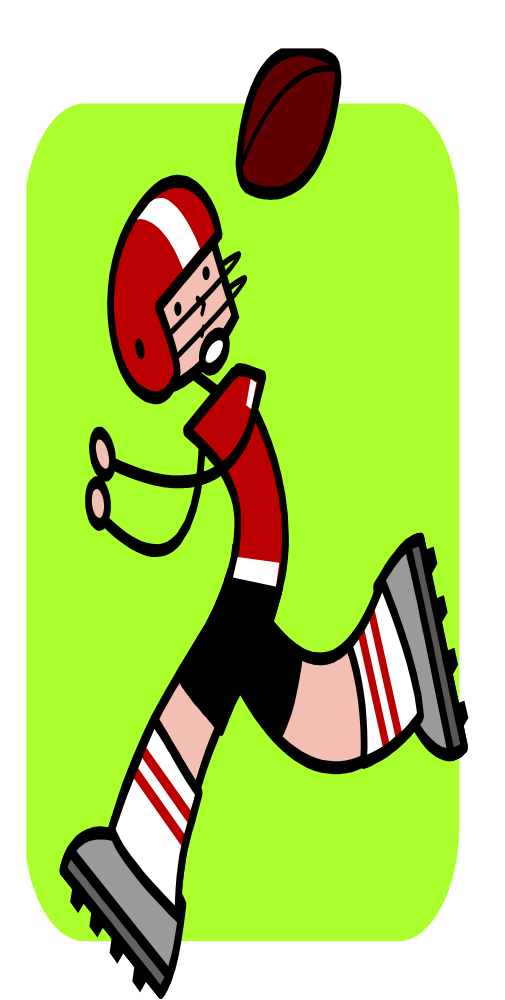

Descriptive data

Table 3 shows the number of DCD children identified by the DCDQ from the group of DCD children identified by the MAND.

\begin{tabular}{l|cccc}
\hline & Mild DCD & Moderate DCD & Severe DCD & \\
\hline MAND & 30 & 9 & 5 \\
DCDQ & 12 & 8 & 5 \\
\hline
\end{tabular}

\section{Conclusion}

A low overall agreement between DCDQ and MAND was obtained in the current study and this is consistent with Green et al.'s (2005) study. With a low test's sensitivity, the DCDQ did not adequately identify DCD in the current sample of Australian children. In addition, the low positive predictive value of the DCDQ brings its clinical utility into question. This suggests that the DCDQ as a screening tool is not highly proficient in discriminating children with DCD from those without DCD. The descriptive data, however, revealed that the DCDQ is accurate in identifying children with moderate to severe DCD. But despite the DCDQ criteria being set at the 'at risk' level, it still did not identify over half of the children with mild DCD.

References

Green, D., Bishop, T., Wilson, B. N., Crawford, S., Hooper, R., Kaplan, B., et al. (2005). Is questionnairebased screening part of the solution to waiting lists for children with developmental coordination disorder? British Journal of Occupational Therapy, 68(1), 2- 10.

McCarron, L. T. (1982). MAND MCCarron assessment of neuromuscular development: Fine and gross motor abilities. Dallas, TX: Common Market Press.

Portney, L. G., \& Watkins, M. P. (2000). Foundations of clinical research: Applications to practice $\left(2^{\text {nd }}\right.$ ed. $)$ New Jersey: Prentice Hall Health

Wilson, B. N. Kaplan, B. J., Crawford, S. G. Campbell, A., \& Dewey, D. (2000). Reliability and validity of a parent questionnaire on childhood motor skills. American Journal of Occupational Therapy, 54, 484-

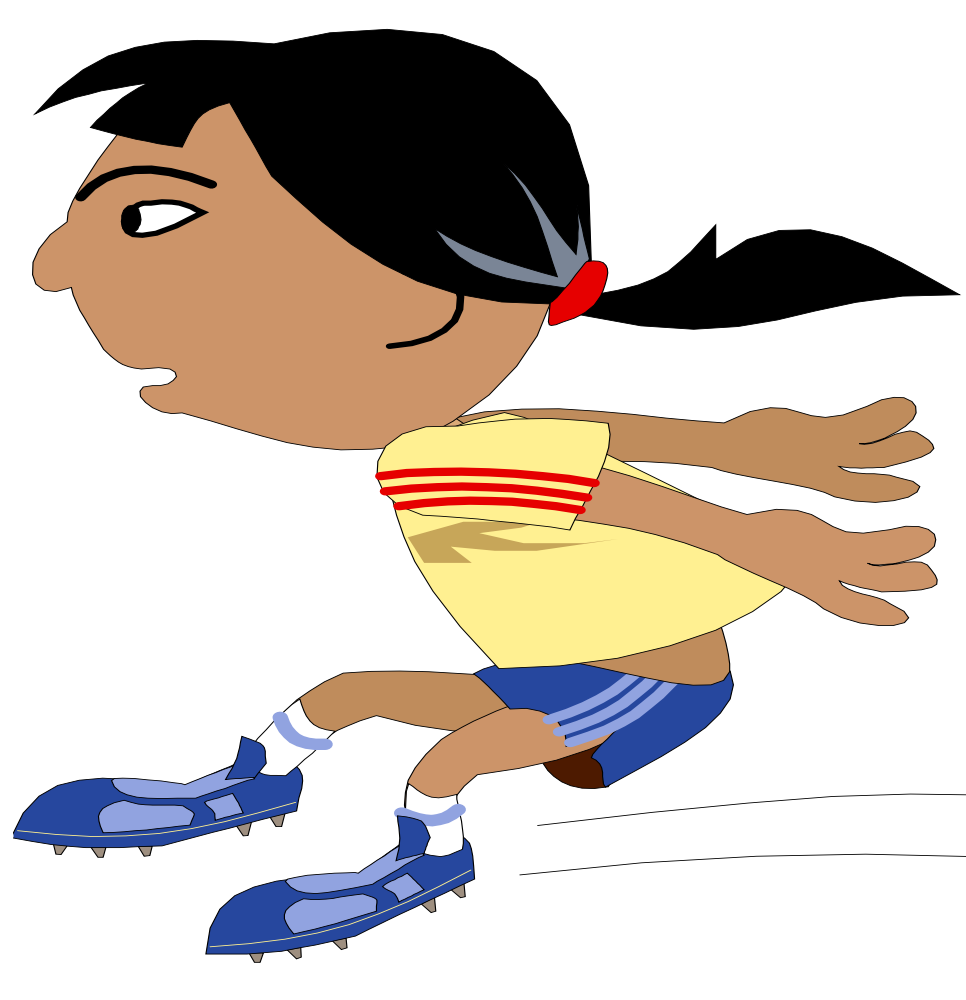

